\title{
Environmental SEM as a Surface-Sensitive Tool to Study Reaction Dynamics
}

Cédric Barroo ${ }^{1,2,3}$, Zhu-Jun Wang ${ }^{3}$ and Marc Georg Willinger ${ }^{3,4}$

1. Chemical Physics of Materials and Catalysis, Université libre de Bruxelles, Belgium.

2. Interdisciplinary Center for Nonlinear Phenomena and Complex Systems (CENOLI), Université libre de Bruxelles, Belgium.

3. Electron Microscopy Group, Department of Inorganic Chemistry, Fritz Haber Institute of the Max Planck Society, Germany.

4. Scientific Center for Optical and Electron Microscopy (ScopeM), ETH Zurich, Switzerland.

Whether we want to study the synthesis, reactivity or ageing of catalytic materials, only experiments performed during the ongoing processes provide data to establish reliable structure-function relationships. Consequently, in the recent years, there has been a growing interest to study catalytic systems in their working state and to develop in situ techniques to approach the experimental conditions encountered in applied formulations of catalysts. Moreover, catalytic applications involve systems composed of active elements with sizes spanning from the $\mathrm{nm}$ - to the mm-scale and a multiscale approach is then necessary to get a more complete picture of the processes at the relevant scales.

In this frame, environmental SEM allows the study of samples with various morphologies, under gas pressures up to hundreds of $\mathrm{Pa}$ and temperatures up to $1500^{\circ} \mathrm{C}$. Recently, this technique has been used to study surface dynamics and the growth of single layers of graphene at different scales $[1,2]$. Indeed, during the formation of graphene via catalytic chemical vapor deposition, the variation in the work function of the substrate and attenuation of the SE emission by even monolayers of graphene is sufficient to be detected. To extend the capabilities of the ESEM technique to the study of gas/solid interactions, the system must be sensitive enough to differentiate work function changes due to gas adsorption. For this, we imaged Pt surfaces during the $\mathrm{NO}_{2}+\mathrm{H}_{2}$ reaction by ESEM. This reaction was previously studied on a single nanoparticle of Pt by field emission techniques [3,4], and the conclusions from these experiments are as follows: adsorbed $\mathrm{NO}_{2}$ species are known to increase the work function of $\mathrm{Pt}$, and nonlinear dynamics can emerge during the reaction, without oxidation of the catalyst. Therefore, if nonlinear behaviors occur during the experiments, it will be a strong indication that surface reaction is happening rather than surface oxidation, which will prove the surface-sensitivity of the ESEM.

Environmental SEM experiments were performed in a modified commercial ESEM (FEI Quanta 200) equipped with a heating stage, gas feeding system and mass spectrometry for analysis of the products of reaction. Pt foils and single crystal were cleaned ex situ, and then annealed under hydrogen environment prior to exposure to reactive mixture of gases. The base pressure of the system corresponds to $\approx 10^{-5} \mathrm{~Pa}$, and then $\mathrm{NO}_{2}$ and $\mathrm{H}_{2}$ gases were injected with mass flow controllers at different pressures ranging from $10^{-3}$ to $20 \mathrm{~Pa}$. Both ETD and LFD detectors were used to follow the reaction dynamics.

The introduction of pure $\mathrm{NO}_{2}$ in the ESEM chamber allows to observe changes in the brightness due to the increasing work function of $\mathrm{Pt}$ in presence of $\mathrm{NO}_{2}$ (Figure 1). After addition of $\mathrm{H}_{2}$, nonlinear behaviors in the form of spiral patterns can be observed (Figure 2) for specific reaction conditions. A brightness analysis of the reaction pattern allows to distinguish 3 different brightness levels on a single propagating wave. This is not only the proof that ESEM is indeed a surface-sensitive technique that can be used to probe gas-surface interactions, but also that ESEM can differentiate different adsorbates 
species as a function of their effect on the work function. Furthermore, it is possible to study the various reaction behaviors, the structure sensitivity of the reaction, the spillover between different facets, and how the presence of boundaries affects the reactivity.

Spiral patterns were observed on both Pt single crystal and Pt foils, on regions as large as $100 \mu \mathrm{m}$ and over four orders of magnitude in pressure. This means that ESEM can be used to observe similar phenomena over different pressure conditions and on samples of increasing complexity, allowing to reduce the pressure and materials gaps. This also demonstrate the robustness of the $\mathrm{NO}_{2}+\mathrm{H}_{2}$ system that can be used as a model reaction to study surface/reactivity relationships [5].

\section{References:}

[1] Z.-J. Wang et al, Nat. Comm. 7 (2016), p. 13256.

[2] M. G. Willinger et al, Microsc. Microanal. 22 (S3) (2016), p. 784.

[3] C. Barroo et al, Appl. Surf. Sci. 304 (2014), p. 2.

[4] C. Barroo et al, Phys. Rev. Lett. 117 (2016), p. 144501.

[5] C. B. thanks the Fonds de la Recherche Scientifique (F.R.S.-FNRS) and the Wallonie-Bruxelles International (Excellence grant WBI.WORLD) for financial support.
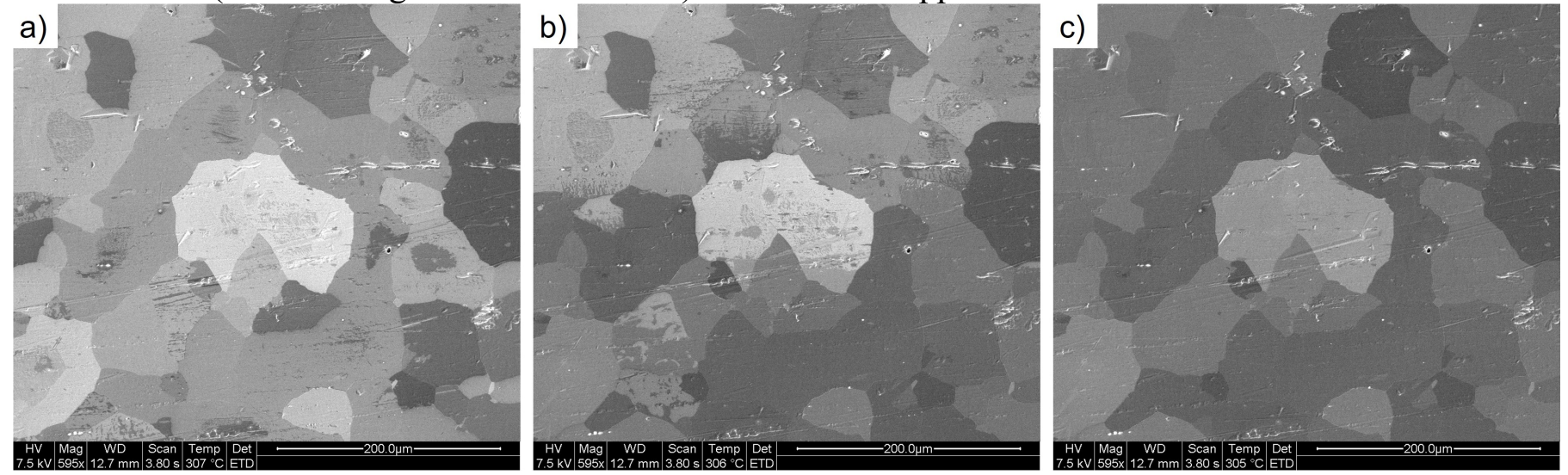

Figure 1. Changes in brightness during the adsorption of $\mathrm{NO}_{2}$ on $\mathrm{Pt}$ foil at $\approx 300^{\circ} \mathrm{C}$.
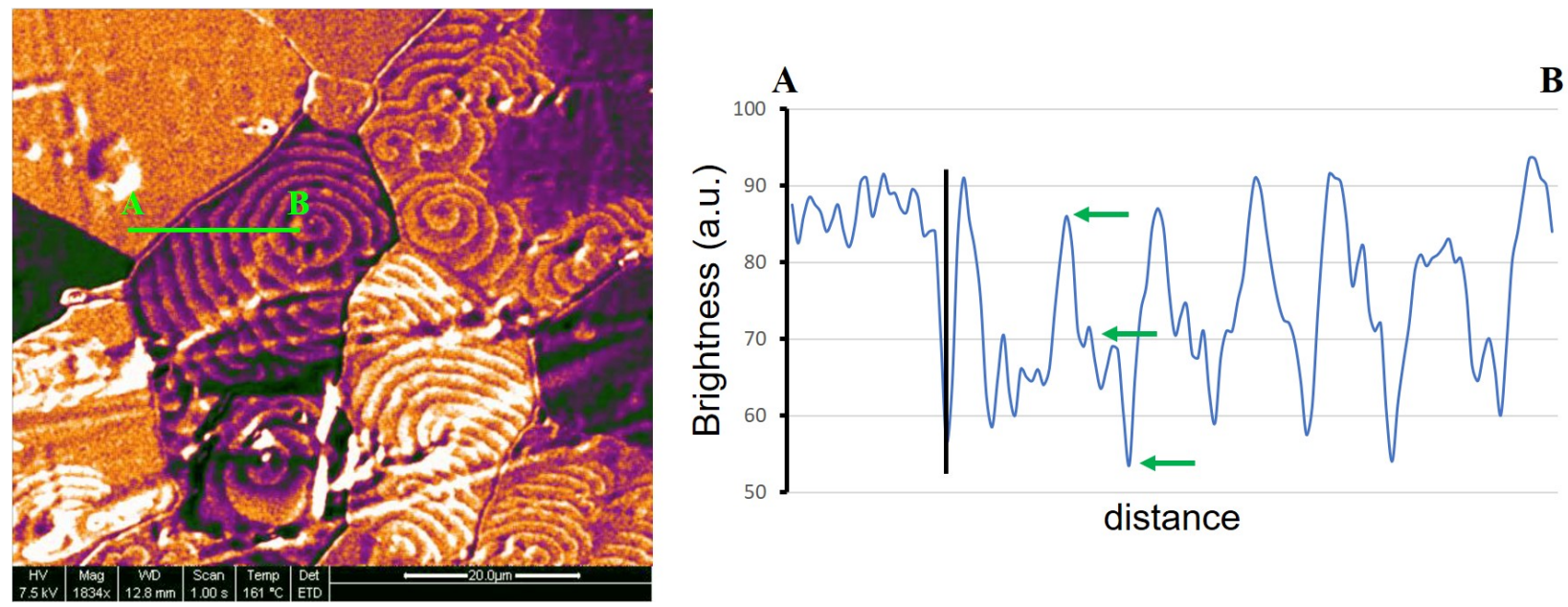

Figure 2. (left) ESEM snapshot during spiral pattern formation in the $\mathrm{NO}_{2}+\mathrm{H}_{2}$ reaction on Pt foil. (right) Brightness analysis showing three distinct brightness levels on a single propagating wave (marked as three arrows) and proving the sensitivity of the ESEM to different adsorbate-species. 